\title{
Is Newer Actually Better? Where Does the 8th Edition Outperform the 7th Edition of the Esophageal TNM Staging System?
}

\author{
Siva Raja, MD, PhD, and Usman Ahmad, MD \\ Department of Thoracic and Cardiovascular Surgery, Cleveland Clinic Foundation, Heart and Vascular Institute, \\ Cleveland, $\mathrm{OH}$
}

Prognostication is one of the fundamental purposes of stage classification. With additional information, each new iteration allows us to refine the stage groupings such that survival within the stage groupings is more similar and survival among stage groupings is more disparate. The 7th edition of the American Joint Committee on Cancer (AJCC) staging manual for esophageal cancer was in and of itself a monumental endeavor that was powered by the World Esophageal Cancer Collaborative (WECC) ${ }^{1}$. WECC-7 was a multi-institutional, multinational collaborative (from 13 institutions from five countries and three continents) that was created for the sole purpose of acquiring data for the creation of the 7th edition staging system. This database accumulated 4627 patients who had undergone an esophagectomy alone for cancer. Several key elements were introduced during this staging system that included (but were not limited to) differentiation by histology, grade of cancer, location, subclassification by number of involved nodes, and further subclassifying T4 into T4a and T4b. Perhaps the most significant contribution was to bring cancers involving the gastroesophageal junction (GEJ) and down to $5 \mathrm{~cm}$ of the stomach into the esophageal staging system, where previously they could have been staged between two different staging systems.

(C) Society of Surgical Oncology 2020

First Received: 24 September 2020

Accepted: 25 September 2020;

Published Online: 22 October 2020

S. Raja, MD, PhD

e-mail: rajas@ccf.org
The 8th edition built on the collaborations started for the 7th edition of the staging manual for esophagus that expanded the WECC to 33 institutions from six continents and included data on 13,300 patients. ${ }^{2}$ While the stage groupings were further refined, the most significant contribution was the creation of pathologic stage groupings for post-induction (yp) patients who had undergone esophagectomy. Previously, the same staging system was being used by clinicians for all patients undergoing esophagectomy, even though it was obvious to most that pT1N0M0 did not have the same survival as ypT1N0M0. In the original work by Sudo et al., the authors explored the prognostic value of the 7th and 8th editions of the AJCC staging manual for patients with esophageal squamous cell cancer undergoing esophagectomy after induction chemotherapy. ${ }^{3}$ They found that for post-induction patients, the 8th edition stage groups had superior prognostic ability.

Since similar analyses led to these stage groupings in the first place, the findings of this study are not unexpected; however, validation through a homogeneous data set is a valuable confirmation. Despite its many strengths, an area of deficiency for data derived from WECC- 8 was that the only endpoint collected was overall survival, and information collected regarding induction therapy only related to chemotherapy, radiotherapy, and chemoradiotherapy. Given the global nature of the database, it is reasonable to expect there was significant variability in the induction protocols. Variability in neoadjuvant therapy was not captured. The true comparison of the 7th and 8th editions can only be made in patients who had not received induction therapy. While not perfectly designed for it, the 7th edition did end up being used for all patients, including those who had undergone neoadjuvant therapy. 
Work is already underway to collect data and plan analyses for the 9th edition of the AJCC staging manual for esophageal cancer. While only time will tell what the finished product might look like, there are many elements that can be added that would add value to the next iteration of this staging system. There are some elements that seem to have already been discussed for inclusion in the 8th edition that had been deferred to the 9th edition; specifically, the placement of GEJ cancers in esophageal adenocarcinomas in an esophageal versus gastric staging schema. In the absence of genetic profiling, this was decided purely by consensus in the 8th edition. Downstaging and residual viable tumor characterization are important predictors of prognosis. ${ }^{4}$ Future iterations will need to continue to separate staging for primary resections versus those after neoadjuvant therapy, but also streamline it for clinical staging where such granular information may not be available.

Oncologic prognostication and guidance of therapy is being heavily shifted towards molecular analyses. Genetic testing may allow diagnosis of the tissue of origin (esophageal vs. gastric), prognosis, and perhaps optimum choice of therapy. As such, expression of markers such as HER2-neu and programmed death ligand-1 (PD-L1) overexpression, as well as mismatch repair (MMR) deficiency, may have therapeutic implications as they have targeted agents now available for treatment. ${ }^{5}$ Lastly, the use and role of 'liquid biopsy' to diagnose and assess tumor burden, and study the effects of neoadjuvant therapy and surveillance after definitive treatment, will continue to evolve. ${ }^{6}$ These and other tumor characteristics that may carry significant prognostic value are likely not mature enough to be part of the 9th edition, but rather be integrated in a future version.

The value of a prognostication schema such as the AJCC staging system is meant to provide a prediction of survival that can be used in a meaningful way by clinicians to guide clinical care. With each iteration, the staging system has been refined for better separation of stage by survival. However, since the 6th edition, each version has included more information and complexity that has made it less 'user friendly' for clinical decision making. This may be inevitable given the ever-increasing number of important variables, and computer algorithms may indeed be needed to risk stratify. Alternatively, with the computing power that is currently present, we may in fact be able to whittle down this long list of variables to a mere handful that can achieve the same result. In the future, we may be able to predict the potential outcome of each patient by treatment modality, and, as such, provide a customized treatment care that transcends our current ideas of staging.

DISCLOSURES Siva Raja and Usman Ahmad have no conflicts of interest with regard to commercial support.

\section{REFERENCES}

1. Rice TW, Blackstone EH, Rusch VW. 7th edition of the AJCC Cancer Staging Manual: esophagus and esophagogastric junction. Ann Surg Oncol. 2010;17(7):1721-1724.

2. Rice TW, Patil DT, Blackstone EH. 8th edition AJCC/UICC staging of cancers of the esophagus and esophagogastric junction: application to clinical practice. Ann Cardiothorac Surg. 2017;6(2):119-130.

3. Sudo N, Ichikawa H, Muneoka Y, et al. Clinical utility of ypTNM stage grouping in the 8th edition of the American Joint Committee on cancer TNM staging system for esophageal squamous cell carcinoma. Ann Surg Oncol. https://doi.org/10.1245/s10434-02009181-3.

4. Raja S, Rice TW, Ehrlinger J, et al. Importance of residual primary cancer after induction therapy for esophageal adenocarcinoma. $J$ Thoracic cardiovasc Surg 2016;152(3):756-761 e755.

5. Raufi AG, Almhanna K. Immune checkpoint inhibitors for esophageal cancer: are we moving in the right direction? Ann Transl Med 2019;7 Suppl 3:S102.

6. Cheung AH, Chow C, To KF. Latest development of liquid biopsy. J Thorac Dis. 2018;10 Suppl 14:S1645-S1651.

Publisher's Note Springer Nature remains neutral with regard to jurisdictional claims in published maps and institutional affiliations. 